\title{
Mirror-less unidirectional radiation in an asymmetric single resonator
}

This paper was downloaded from TechRxiv (https://www.techrxiv.org).

\section{LICENSE}

CC BY-NC-SA 4.0

SUBMISSION DATE / POSTED DATE

20-07-2021 / 22-07-2021

CITATION

Kim, Sangin; Song, Joohyung; Heo, Hyungjun; Lee, Sangjun (2021): Mirror-less unidirectional radiation in an asymmetric single resonator. TechRxiv. Preprint. https://doi.org/10.36227/techrxiv.15020034.v1

DOI

10.36227/techrxiv.15020034.v1 


\title{
Mirror-less unidirectional radiation in an asymmetric single resonator
}

\author{
Joohyung Song, Hyungjun Heo, Sangjun Lee and Sangin Kim
}

\begin{abstract}
We propose a novel approach to realize unidirectional radiation in an asymmetric single resonator without using an external mirror, which is based on mutual coupling between two degenerate resonant modes through the background scattering of the asymmetric resonator. In the proposed approach, only one of the resonant modes experiences optical gain and the other works just as an internal reflector. So, our approach is conceptually to mimic a one-port system by introducing the internal reflector in a two-port system. Due to this intuitive and simple operation concept, the design of such devices is straightforward. We described the operation principle by using the temporal coupled-mode theory, from which a practical design method was derived. An example device of the proposed approach was designed based on guided-mode resonances in a slab waveguide grating and its performance was analyzed with the finite-difference time-domain simulation. A ratio of $4.6 \times 10^{4}$ between the upward and the downward radiations was numerically demonstrated.
\end{abstract}

Index Terms - Unidirectional radiation, surface emitting lasers, zero-contrast gratings, guided-mode resonance

\section{INTRODUCTION}

$\mathrm{I}_{\mathrm{s}}$ $\mathrm{n}$ an asymmetric single-mode resonator of two-port (two external decay paths) such as a one- or two-dimensional photonic crystal slab, the ratio between two decay rates cannot exceed $(1+r) /(1-r)$, where $r$ is the reflection of the background scattering [1]. This implies that the asymmetric decay rate ratio can be infinite only when $r=1$. However, the background scattering with $r=1$ is not physically feasible, so that the singlemode resonator cannot have an infinite asymmetric decay rate ratio. Therefore, when a light source such as a laser or a light emitting diode (LED) is implemented based on the two-port single-mode resonator, unidirectional emission is fundamentally impossible without using an external mirror. Obviously, the usage of the external mirror such as a distributed Bragg reflector (DBR) or a metal reflector in the light source has many drawbacks. The DBR requires a sophisticated epitaxial growth process, and moreover, a high performance

This work was supported by National Research Foundation of Korea (2020R1A2B5B01002681，2021R1A4A1033155). This work was also supported by the GRRC program of Gyeonggi province (GRRC-AJOU-2016B01, Photonics-Medical Convergence Technology Research Center).
DBR is available only in a certain material system, i.e., GaAs/ $/ \mathrm{Al}_{\mathrm{x}} \mathrm{Ga}_{1-\mathrm{x}} \mathrm{As}$. This limits the range of the emission wavelength of such a light source based on a monolithically grown wafer or requires a complicated fabrication process such as wafer bonding to implement a freely chosen emission wavelength $[2,3]$. In order to employ the metal reflector, an additional substrate removal or thinning process is needed.

Recently, H. Zhou et al. has shown that mirror-less singlesided radiation is achievable in a one-dimensional photonic crystal slab of broken $C_{2}^{z}$ symmetry, in which the broken $C^{z_{2}}$ symmetry makes two opposite-direction propagating modes with nonzero in-plane momentum $\left(\vec{k}_{\|} \neq 0\right)$ distinguishable and the interaction between the two degenerate resonant modes enables to overcome the limit of the single-mode resonator [4]. Since their structure works for oblique incoming/outgoing waves, it was treated as a four-port system. Moreover, since the $C^{z}{ }_{2}$ symmetry of the structure is broken, its design and fabrication process will be rather complicated.

In this work, a novel approach to implement unidirectional radiation from a single resonator without an external mirror is proposed, which is based on mutual coupling between two degenerate resonant modes through the background scattering of the asymmetric resonator. In the proposed approach, only one of the resonant modes is responsible for emission, and the other works just as an internal mirror in conjunction with the background scattering. So, conceptually, the proposed approach can be understood as mimicking one-port resonant system due to the internal mirror function. In our previous work, we have shown that a flat-top broadband reflector can be realized by combining a single guided-mode resonance (GMR) and Fabry-Perot (F-P) like background scattering in a slab waveguide grating, and $100 \%$ reflection occurs when the GMR coincides with the resonance of the F-P like background scattering $(r=0$ or $t=1)$ [5]. If another resonant mode of the same resonance frequency with an optical gain is introduced in this $100 \%$ reflector structure, we may get unidirectional emission due to the internal reflection function. This is the basic concept of our approach, which is quite simple and intuitive. However, the interaction among two resonant modes and the background scattering is not fully considered in this apparently oversimplified approach. So, in section 2, the operation

Joohyung Song, Sangjun Lee and Sangin Kim are with the Department of Electrical and Computer Engineering, Ajou University, Suwon 16499, South Korea. (e-mail: lilatop@ajou.ac.kr; 1sjkmjh@ajou.ac.kr; sangin@ajou.ac.kr).

Hyungjun Heo is with Center for Quantum Information, Korea Institute of Science and Technology (KIST), Seoul 02792, South Korea. (e-mail: hyungjun.heo@kist.re.kr). 
principle and design requirement of our unidirectional emitter is theoretically described with the temporal coupled-mode theory (TCMT) [6], proving that our simple and intuitive approach really works. In section 3 , the theory is confirmed by an example design of a unidirectional surface emitter composed of a vertically asymmetric slab waveguide grating, dubbed as a zero-contrast grating (ZCG) [7], supporting two GMR modes. In our proposed approach, no specific structural symmetry is required, and thus, the designed device structure is easy to fabricate, requiring only a single etching process. Another distinguishing factor of our approach is that the direction of emission is normal to the slab. The performance of the designed device is analyzed with the commercial finite-difference timedomain (FDTD) simulation (Lumerical).

\section{THEORETICAL DESCRIPTION OF THE UNIDIRECTIONAL EMITTER}

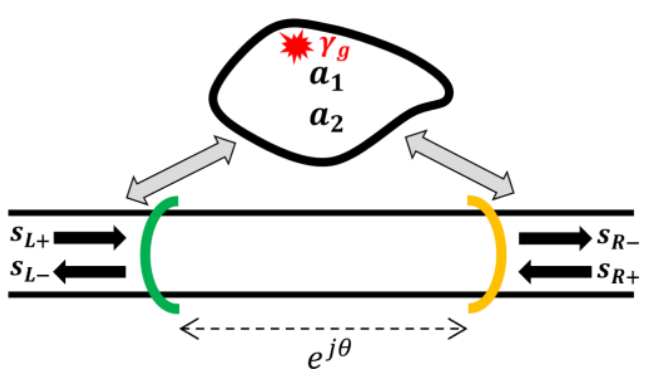

Fig. 1. Schematic of a two-port system composed of an asymmetric single resonator with two resonant modes and a wave propagation channel with partial reflections.

In this section, we formulate the TCMT to deal with an asymmetric optical resonator of two resonant modes $\left(a_{1}, a_{2}\right)$ coupled to two external ports (L- and R-port) as shown in Fig 1. The waveguide-like structure in Fig. 1 represents the wave propagation channel through which the resonant modes and the external ports are coupled. In this work, it is assumed that there are two partial reflections in the channel, which results in the background scattering. The dynamic equation of amplitudes $\left(a_{1}, a_{2}\right)$ of two resonant modes can be described as [6]

$$
\begin{gathered}
\frac{d A}{d t}=\left(j \Omega_{0}-\Gamma+\mathrm{G}\right) A+\mathrm{K}^{T}\left(\begin{array}{l}
S_{L+} \\
s_{R+}
\end{array}\right), \\
\text { where } A=\left(\begin{array}{c}
a_{1} \\
a_{2}
\end{array}\right), \Omega_{0}=\left(\begin{array}{cc}
\omega_{1} & 0 \\
0 & \omega_{2}
\end{array}\right), \\
\Gamma=\left(\begin{array}{cc}
\gamma_{1} & \mu_{12} \\
\mu_{21} & \gamma_{2}
\end{array}\right), G=\left(\begin{array}{cc}
\gamma_{g} & 0 \\
0 & 0
\end{array}\right), \mathrm{K}=\left(\begin{array}{cc}
\kappa_{L 1} & \kappa_{L 2} \\
\kappa_{R 1} & \kappa_{R 2}
\end{array}\right) .
\end{gathered}
$$

$A$ is a $2 \times 1$ vector of the resonant mode amplitudes, where $a_{i}$ is normalized such that $\left|a_{i}\right|^{2}$ represents the energy stored in $i$-th mode. $\Omega_{0}$ is a diagonal matrix, where $\omega_{i}$ is a real value and represents the resonant frequency of the $i$-th mode. $s_{L+}$ and $s_{L^{-}}\left(s_{R^{+}}\right.$and $\left.s_{R^{-}}\right)$are the amplitudes of incoming and outgoing waves at L-port (R-port), which are normalized such that $|s|^{2}$ represents the power carried by the wave. $\Gamma$ describes the decay and mutual coupling property of the resonant modes. $\gamma_{i}$ is the decay coefficient of the $i$-th mode. $\mu_{12}$ is the mutual coupling coefficient from $a_{2}$ to $a_{1}$ and vice versa for $\mu_{21}$. In a single resonator system, the resonant modes are orthogonal, so that there is no direct mutual coupling between the modes. However, due to the partial reflectivity in the channel, the resonant modes are indirectly coupled though the channel, which is described by $\mu_{i j}$. If we consider the coupling behavior between the resonant mode and the propagating waves inside the channel cavity formed by the partial reflectivity assuming no incoming waves, it is shown that the mutual coefficients are the same i.e., $\mu_{12}=\mu_{21}=\mu$, which is derived in Appendix. In Appendix, it is also shown that the decay rates $\left(\gamma_{1}\right.$ and $\left.\gamma_{2}\right)$ and the mutual coupling coefficient $(\mu)$ are complex values in general. This implies that the mutual coupling through the partial reflectivity in the channel can also result in the resonant frequency change in each mode, that is, an effective resonance frequency becomes $\left(\omega_{i}+j \frac{\gamma_{i}-\gamma_{i}^{*}}{2}\right)$. G represents an optical gain, and it is assumed that only mode $1\left(a_{1}\right)$ is excited with a gain rate $\left(\gamma_{g}\right)$ of a real value. $\mathrm{K}$ is the coupling coefficient matrix between resonant modes and input $\left(s_{L_{+}}, s_{R+}\right)$ where $\kappa_{p q}(p: \mathrm{L}, \mathrm{R}, q: 1,2)$ indicates the coupling coefficient of $p$-port and $q$-th mode.

Considering the background scattering due to the partial reflectivity in the channel coupled to the external ports, the outgoing waves are related to the incoming waves and the mode amplitudes as

$$
\begin{aligned}
& \left(\begin{array}{l}
s_{L-} \\
s_{R-}
\end{array}\right)=C\left(\begin{array}{l}
s_{L+} \\
s_{R+}
\end{array}\right)+D A, \\
& \text { where } C=e^{j \theta}\left(\begin{array}{cc}
r & j t \\
t & r
\end{array}\right) ;|r|^{2}+|t|^{2}=1, D=\left(\begin{array}{ll}
d_{L 1} & d_{L 2} \\
d_{R 1} & d_{R 2}
\end{array}\right) .
\end{aligned}
$$

$C$ is the background scattering matrix with a reflection coefficient $r$ and a transmission coefficient $t$. $\theta$ is the phase retardation between $\mathrm{L}-$ and $\mathrm{R}$-ports. $\mathrm{D}$ is the coupling coefficient matrix between resonant modes and output waves $\left(s_{L-}, s_{R-}\right)$ where $d_{p q}(p: \mathrm{L}, \mathrm{R}, q: 1,2)$ indicates the coupling coefficient of $p$-port and $q$-th mode.

The system described by (1) and (2) should satisfy the energy conservation and time-reversal symmetry when there is no gain $(G=0)$ [8]. From the energy conservation, $D$ and $\Gamma$ are related as

$$
D^{\dagger} D=\Gamma^{\dagger}+\Gamma .
$$

From the time reversal symmetry, we can derive the relations between $\mathrm{K}, \mathrm{D}$ and $C$ as

$$
\begin{gathered}
C D^{*}=-D \\
K=D .
\end{gathered}
$$

The relations among the coupling and the decay coefficients are associated with the background scattering characteristics through (3) and (4). In this work, $r=0$ is assumed. The condition of $r=0$ or $t=1$ (the resonance of the F-P like background scattering) is also required in the approach suggested by $\mathrm{H}$. Zhou et al. [4]. Note that $r=0$ does not mean $\mu=0$. The existence of the partial reflectivity in the channel results in a non-zero mutual coupling coefficient as discussed in Appendix, and $r=0$ occurs when $\theta=m \pi$ ( $\mathrm{m}$ is an integer.) $[4,5]$. When $r=0$ is assumed, from (4) and (5), the coupling coefficients of each mode into the respective ports are related as

$$
\begin{aligned}
& d_{R 1}=e^{j\left(\theta-\frac{\pi}{2}\right)} \cdot d_{L 1}^{*}, \\
& d_{R 2}=e^{j\left(\theta-\frac{\pi}{2}\right)} \cdot d_{L 2}^{*} .
\end{aligned}
$$


Substituting (6) into (3), we obtain

$$
\begin{gathered}
\left|\mathrm{d}_{\mathrm{L} 1}\right|^{2}=\left|\mathrm{d}_{R 1}\right|^{2}=\frac{\gamma_{1}+\gamma_{1}^{*}}{2}, \\
\left|d_{L 2}\right|^{2}=\left|d_{R 2}\right|^{2}=\frac{\gamma_{2}+\gamma_{2}^{*}}{2}, \\
d_{L 1} \cdot \mathrm{d}_{L 2}^{*}+d_{R 1} \cdot \mathrm{d}_{R 2}^{*}=\mu+\mu^{*} .
\end{gathered}
$$

(7a) and (7b) imply that when $r=0$, each resonant mode emits the same amount of energy into the respective ports, and the magnitude of the coupling coefficient of each mode is the same as the real part of the decay rate. By dividing (6a) by (6b), the phases of the coupling coefficients are related as

$$
\varphi_{R 1}-\varphi_{R 2}=-\left(\varphi_{L 1}-\varphi_{L 2}\right)
$$

where $\varphi_{R 1,2}$ and $\varphi_{L 1,2}$ are the phases of $\mathrm{d}_{R 1,2}$, and $\mathrm{d}_{L 1,2}$, respectively.

\section{A. Conditions for the unidirectional emission}

In order to find the lasing conditions, we solved (1) in a steady state assuming no incoming waves $\left(s_{L+}=s_{R+}=0\right)$. In the steady-state, (1) becomes

$$
\left(\begin{array}{cc}
j\left(\omega_{o}-\omega_{1}\right)-\left(\gamma_{g}-\gamma_{1}\right) & \mu \\
\mu & j\left(\omega_{o}-\omega_{1}\right)+\gamma_{2}
\end{array}\right) A=0,
$$

where $\omega_{o}$ is a lasing frequency. (9) can have a non-trivial solution only when

$$
\operatorname{det}\left(\begin{array}{cc}
j\left(\omega_{o}-\omega_{1}\right)+\left(\gamma_{1}-\gamma_{g}\right) & \mu \\
\mu & j\left(\omega_{o}-\omega_{2}\right)+\gamma_{2}
\end{array}\right)=0 .
$$

When lasing occurs, from (9), the amplitudes of the modes are related as

$$
a_{2}=-\frac{\mu}{j\left(\omega_{0}-\omega_{2}\right)+\gamma_{2}} a_{1} .
$$

Substitution of (11) into (2) with $\left(s_{L+}=s_{R+}=0\right)$ gives us the normalized outgoing wave amplitude at the respective port:

$$
\begin{aligned}
& \text { at L-port, } \frac{s_{L-}}{a_{1}}=d_{L 1}-\frac{\mu \cdot d_{L 2}}{j\left(\omega_{o}-\omega_{2}\right)+\gamma_{2}}, \\
& \text { at R-port, } \frac{s_{R-}}{a_{1}}=d_{R 1}-\frac{\mu \cdot d_{R 2}}{j\left(\omega_{o}-\omega_{2}\right)+\gamma_{2}} .
\end{aligned}
$$

So, the unidirectional emission condition is achieved by making either of (12a) or (12b) equal to zero. For physically meaningful unidirectional emitter implementation, only one of (12a) and (12b) should be zero, avoiding the case that makes (12a) and (12b) zero simultaneously. For instance, to achieve unidirectional emission into L-port, following conditions should be satisfied:

$$
d_{L 1}-\frac{\mu \cdot d_{L 2}}{j\left(\omega_{o}-\omega_{2}\right)+\gamma_{2}} \neq 0
$$

and

$$
d_{R 1}-\frac{\mu \cdot d_{R 2}}{j\left(\omega_{o}-\omega_{2}\right)+\gamma_{2}}=0 .
$$

Substitution of (13b) into (13a) gives us

$$
d_{L 1}-\frac{d_{R 1} \cdot d_{L 2}}{d_{R 2}} \neq 0 .
$$

Now, (14) can be further simplified with the assumption of $r=$ 0 . Using (6), (14) is turned into

$$
e^{j\left(\varphi_{L 1}-\varphi_{L 2}\right)}-e^{j\left(\varphi_{R 1}-\varphi_{R 2}\right)} \neq 0 .
$$

From (8), one can see that (15) is equivalent to

$$
\varphi_{R 1}-\varphi_{R 2} \neq 0 \text {. }
$$

As a result, the conditions for the unidirectional emission into L-port can be summarized as (13b) and (16) with $r=0$. By the way, these conditions are not enough to specify all the coefficients, which implies that we still have degrees of freedom in choosing those coefficients to implement the unidirectional emission. So, we also have a degree of freedom to choose the lasing frequency satisfying (10). It is a reasonable to assume that lasing occurs at the adjusted (or effective) resonance frequency of mode $1\left(\omega_{o}=\omega_{1}+j \frac{\gamma_{1}-\gamma_{1}^{*}}{2}\right)$. This assumption is used in the following discussion and will be justified later.

\section{B. Conditions for the passive virtual one-port system}

The condition for the unidirectional emission derived in the previous section is rather abstract at this moment. To obtain physical meaning of the condition and to get the idea how to implement it, in this section, we consider the passive asymmetric two-port system mimicking a one-port system. We are going to derive the condition to obtain perfect reflection with single-sided illumination in the same optical system of no optical gain in conjunction with the condition for the unidirectional laser, showing that these conditions can be simultaneously satisfied. This implies that the asymmetric twomode resonator based perfect reflector will also work as the unidirectional laser when a proper optical gain is introduced to one of the resonant modes.

The whole system is the same as the one considered in the previous section except that there is no optical gain. We consider single-sided illumination from L-port $\left(s_{L+} \neq\right.$ $\left.0, s_{R+}=0\right)$. At the steady state, from (1), the mode amplitudes $\left(a_{1}\right.$ and $\left.a_{2}\right)$ are related to $s_{L+}$ at an operating frequency of $\omega$ as follows:

$$
\begin{aligned}
& a_{1}=\left(\frac{-\mu \cdot d_{L 2}+\left(j\left(\omega-\omega_{2}\right)+\gamma_{2}\right) \cdot d_{L 1}}{\left(j\left(\omega-\omega_{1}\right)+\gamma_{1}\right) \cdot\left(j\left(\omega-\omega_{2}\right)+\gamma_{2}\right)-\mu^{2}}\right) s_{L+}, \\
& a_{2}=\left(\frac{-\mu \cdot d_{L 1}+\left(j\left(\omega-\omega_{1}\right)+\gamma_{1}\right) \cdot d_{L 2}}{\left(j\left(\omega-\omega_{1}\right)+\gamma_{1}\right) \cdot\left(j\left(\omega-\omega_{2}\right)+\gamma_{2}\right)-\mu^{2}}\right) s_{L+} .
\end{aligned}
$$

From (2), the outgoing wave at R-port is given by

$$
s_{R-}=j t \cdot e^{j \theta} \cdot s_{L+}+d_{R 1} \cdot a_{1}+d_{R 2} \cdot a_{2} .
$$

Substituting (17a) and (17b) into (18), we obtain a transmission coefficient as

$$
T=\frac{s_{R-}}{s_{L+}}=j t \cdot e^{j \theta}+
$$

$\frac{-\mu \cdot d_{R 1} \cdot d_{L 2}+\left(j\left(\omega-\omega_{2}\right)+\gamma_{2}\right) \cdot d_{R 1} \cdot d_{L 1}-\mu \cdot d_{L 1} \cdot d_{R 2}+\left(j\left(\omega-\omega_{1}\right)+\gamma_{1}\right) \cdot d_{L 2} \cdot d_{R 2}}{\left(j\left(\omega-\omega_{1}\right)+\gamma_{1}\right) \cdot\left(j\left(\omega-\omega_{2}\right)+\gamma_{2}\right)-\mu^{2}}$.

The perfect reflection (zero transmission, $T=\frac{s_{R-}}{s_{L+}}=0$ ) at the chosen lasing frequency of the unidirectional emitter $\left(\omega_{o}=\right.$ $\omega_{1}+j \frac{\gamma_{1}-\gamma_{1}^{*}}{2}$ ) requires

$$
|\mu|^{2}=\frac{\gamma_{1}+\gamma_{1}^{*}}{2} \cdot \frac{\gamma_{2}+\gamma_{2}^{*}}{2},
$$

where (13b), (7), (8) and the assumption of $r=0(\leftrightarrow t=1)$ are used. From (20), using (7c) and (8), we obtain

$$
\mu=\sqrt{\frac{\gamma_{1}+\gamma_{1}^{*}}{2} \cdot \frac{\gamma_{2}+\gamma_{2}^{*}}{2}} \cdot e^{j\left(\varphi_{R 1}-\varphi_{R 2}\right)} .
$$


Substituting (21), which is the condition to mimic one-port system at the chosen lasing frequency, and (6) into (13b), which is the unidirectional emission condition, we obtain

$$
\omega_{o}=\omega_{1}+j \frac{\gamma_{1}-\gamma_{1}^{*}}{2}=\omega_{2}+j \frac{\gamma_{2}-\gamma_{2}^{*}}{2} .
$$

This implies that the effective resonance frequencies of mode 1 and 2 should be the same, that is, two resonant modes should be degenerate with the mode coupling effect due to the background scattering, i.e, the partial reflectivity in the propagation channel, considered. So, if the two-port asymmetric optical resonator of two resonant modes is designed to satisfy (16), (21), (22), and $r=0$, it works as the perfect reflector at the lasing frequency when there is no optical gain, and also works as the unidirectional emitter when a proper optical gain is introduced to one of the resonant modes.

By the way, the phase of the mutual coefficient in (22) is not specified yet, which can be found by considering the proper optical gain for lasing operation. The proper value of gain needed for lasing is determined by (10) and (22), which results in

$$
\left(\frac{\gamma_{1}+\gamma_{1}^{*}}{2}-\gamma_{g}\right) \cdot\left(\frac{\gamma_{2}+\gamma_{2}^{*}}{2}\right)=\mu^{2}
$$

Substituting (21) into (23), we obtain

$$
\gamma_{g}=\frac{\gamma_{1}+\gamma_{1}^{*}}{2} \cdot\left(1-e^{j 2\left(\varphi_{R 1}-\varphi_{R 2}\right)}\right)
$$

The requirement that the gain $\left(\gamma_{g}\right)$ should be a positive real value, which results in

$$
\begin{gathered}
\varphi_{R 1}-\varphi_{R 2}= \pm \frac{\pi}{2} \\
\gamma_{g}=\gamma_{1}+\gamma_{1}^{*} .
\end{gathered}
$$

Note that (25) does not conflict with (16). (26) implies that mode 2 combined with the background scattering due to the partial reflection in the channel only works as a perfect reflector for the unidirectional emitter. More importantly, substitution (25) into (21) gives us

$$
\mu= \pm j \sqrt{\frac{\gamma_{1}+\gamma_{1}^{*}}{2} \cdot \frac{\gamma_{2}+\gamma_{2}^{*}}{2}}
$$

Therefore, the final design conditions are summarized as (22), (25), (27), and $r=0$, which provide a virtual (mimicking) oneport resonator system for both the passive and the active (lasing) operations.

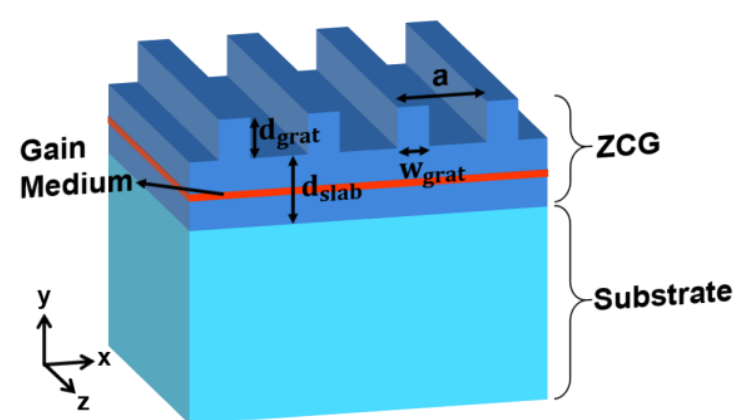

Fig. 2. Designed unidirectional surface emitting laser based on ZCG. A ZCG of $n_{\mathrm{ZCG}}=3.45$ is placed above the substrate of $n_{\text {Sub }}=1.6$. A thin layer of gain medium is embedded in the ZCG. The designed optimal parameters are $a=$ $570 \mathrm{~nm}, d_{\text {Slab }}=336 \mathrm{~nm}, d_{\text {Grat }}=203 \mathrm{~nm}$, and $F F=w_{\text {grat }} / a=0.353$.

\section{DESIGN OF UNIDIRECTIONAL EMITTER}

Based on the theoretical analysis described in the previous section, we designed a unidirectional emitter that mimics oneport resonant system. Fig. 2 shows the designed unidirectional emitter which consists of a ZCG $\left(n_{\mathrm{ZCG}}=3.45\right)$ stacked on a substrate $\left(n_{\text {Sub }}=1.6\right)$. In numerical analysis, for simplicity, it is assumed that the thin layer of gain medium embedded in the ZCG has the same refractive index as the ZCG. A fill factor $(F F)$ is defined as $w_{\text {Grat }} / a$. In this work, transverse electric (TE) polarized wave $\left(H_{\mathrm{x}}, H_{\mathrm{y}}, E_{\mathrm{z}} \neq 0\right)$ is considered.

The ZCG, which is a vertically asymmetric resonator, can have numerous GMR modes mainly depending on the slab thickness $\left(d_{\text {slab}}\right)$ [7], and the partial reflectivity at two surfaces of ZCG brings about the F-P like background scattering. As derived in the previous section, to design a virtual one-port resonant system of two resonant modes, we should optimize the structural parameters to obtain two degenerate GMR modes having a proper interacting (coupling) strength due to the background scattering. In addition, the background scattering itself should satisfy the resonant condition $(r=0$ or $t=1)$ at the resonant frequency of those two modes. In the design of the structure, we first designed a broadband reflector of $100 \%$ peak reflectivity based on the interaction between a relatively low quality factor $(\sim 10)$ GMR mode and the background scattering, where $\sim 100 \%$ peak reflectivity verifies the coincidence of the resonance frequencies of the low quality factor GMR mode and the background scattering [5]. Then, the structure is further optimized to make the resonant frequency of another GMR mode of a relatively high quality factor $(>\sim 1000)$ coincide with the peak reflectivity frequency of the broadband reflector. In the second optimization process, when the interaction strength between those two GMR is balanced with the geometric mean of their decay rates (see (27)), the degeneracy of those two GMR gives us a Gaussian-like symmetric reflection spectrum. Otherwise, the resulting reflection spectrum shows the Fano resonance shape.

Fig. 3(a) show the reflectivity map as a function of $d_{\text {slab }}$ with other structural parameters fixed to the optimal values of our design for $\lambda=934.9 \mathrm{~nm}$; period $a=570 \mathrm{~nm}, d_{\text {Grat }}=203 \mathrm{~nm}, F F$ $=0.353$. In the calculation of the reflection spectrum, the rigorous coupled wave analysis (RCWA) [8] was used. One can see that two reflection peak branches intersect near the slab thickness of $d_{\text {Slab }}=336 \mathrm{~nm}$, where a symmetric broadband reflection spectrum with a peak reflectivity of $\sim 100 \%$ is achieved as shown in Fig, 3(b). The branch of a broad bandwidth originates from a low quality factor GMR combined with the F-P like background scattering [5]. The electric field $\left(\left|E_{z}\right|\right)$ profiles at the reflection peak points for two different slab thicknesses of $d_{\text {Slab }}=320 \mathrm{~nm}$ and $360 \mathrm{~nm}$ are plotted in the insets of Fig. 3(a), which verifies that the broadband reflection branch originates from the same single GMR of the first diffraction order. Whereas, for $d_{\text {Slab }}=336 \mathrm{~nm}$, which is of the optimal design, the electric field profile at the reflection peak shows mainly the high quality factor GMR mode shape. In this case, although the normally incident wave excites those two GMR modes simultaneously, the energy portion stored in the high quality factor GMR mode is way much larger due to the huge difference in the quality factor between those two GMR modes, and thus, the normalized field profile is dominantly represented 
by the high quality factor mode. From the field profile, one can also note that the high quality factor GMR mode stems from the second-order diffraction and its field is strongly confined in the slab region. So, if the thin gain medium layer is deliberately placed at the zero field (nodal) plane of the low quality factor GMR mode, only the high quality factor GMR mode experiences the optical gain, and the low quality factor mode combined with the background scattering will serve as the reflector as the TCMT described.
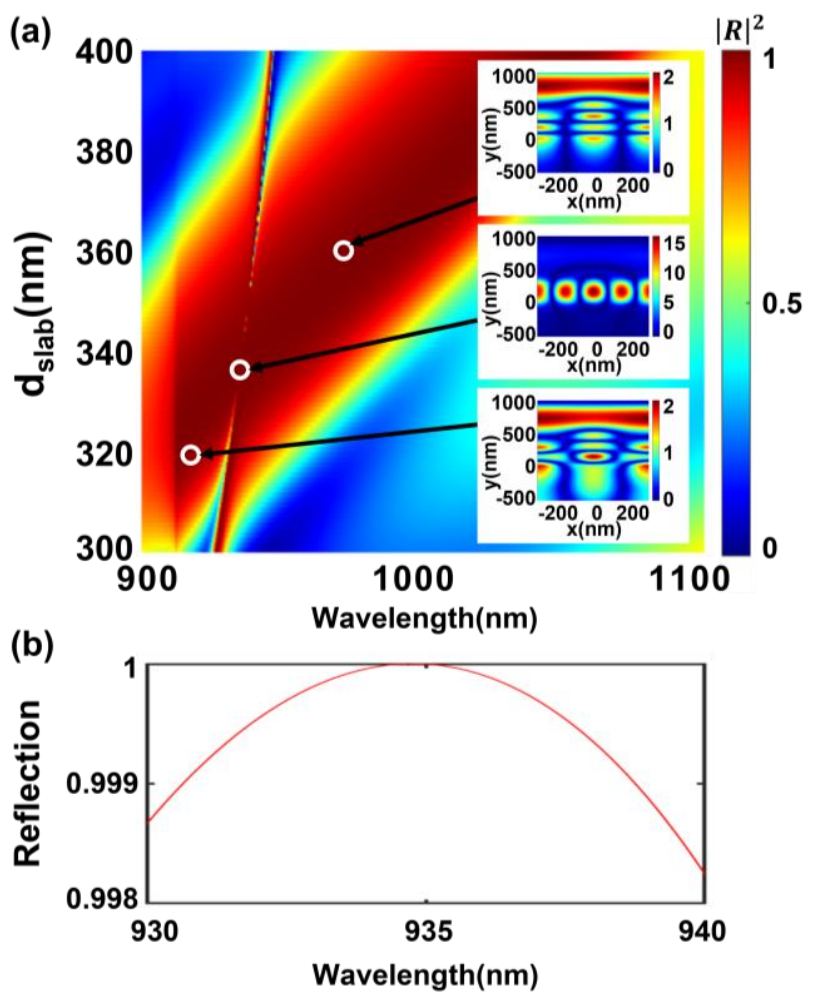

Fig. 3. (a) Reflection map as a function of slab thickness for optimal structure without gain. Inset: the normalized electric field at different reflection peak points for $d_{\text {Slab }}=320 \mathrm{~nm}, 336 \mathrm{~nm}$ and $360 \mathrm{~nm}$. (b) Reflection spectrum for the optimal slab thickness of $d_{\text {Slab }}=336 \mathrm{~nm}$.

The performance of the designed unidirectional laser was analyzed with the FDTD simulation. First, we calculated the photonic band structure of the designed device with FDTD simulation to confirm the GMR modes involved in the unidirectional laser operation, which is plotted in Fig. 4(a). In the photonic band structure calculation, many broadband dipole sources of TE polarization located off the symmetry lines in the ZCG were used to excite all the resonant modes, and the spectra were calculated for various $k_{\|}$values with the Bloch boundary condition applied in the horizontal direction and the absorbing (perfectly matched layer) boundary condition applied in the vertical direction [9]. In the spectrum calculation for each $k_{\|}$ value, the spectra calculated from the fields at various locations in the ZCG, away from the sources, were averaged to eliminate the dependency on the field monitoring position. The calculation time was set just long enough to obtain stabilized spectra. If the calculation time is too long, the spectral component of the low quality factor mode disappears. In the frequency range of our interest, three resonant mode branches are observed; the high and the low quality factor GMR modes as observed in Fig. 3(a) and a bound state in the continuum which disappears for $k_{\|}=0$ and thus, is not involved in the radiation into the normal direction of our designed laser. Fig. 4(b) shows the calculated spectrum for $k_{\|}=0$ and the insets show the electric field $\left(\left|\mathrm{E}_{\mathrm{z}}\right|\right)$ profiles near the resonance frequency ( $\mathrm{f}=0.6095 \mathrm{c} / \mathrm{a}$ corresponding to $\lambda=934.9 \mathrm{~nm}$ ), which are the same as the RCWA calculation shown in Fig. 3(a) and confirms the degeneracy of the two GMR modes.
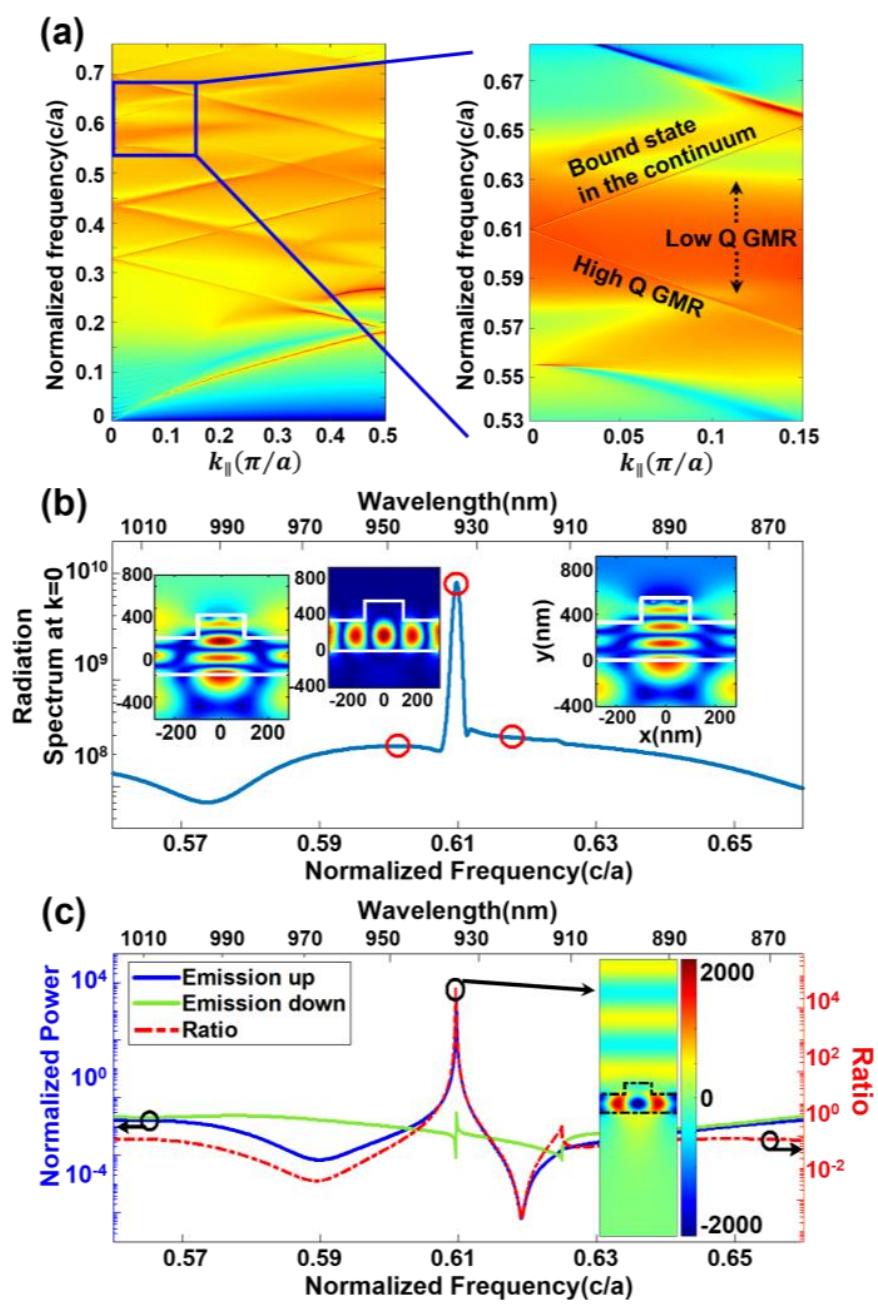

Fig. 4. (a) Photonic band structure (TE polarization) of the designed unidirectional laser calculated with the FDTD simulation. (b) Averaged radiation spectrum calculated for $k_{\|}=0$. In the insets, mode profiles $\left(\left|\mathrm{E}_{\mathrm{z}}\right|\right)$ of two GMR modes involved in the unidirectional lasing operation are plotted; the low quality factor mode profiles at $\mathrm{f}=0.6042 \mathrm{c} / \mathrm{a}$ and $0.6175 \mathrm{c} / \mathrm{a}$, and the high quality factor mode profile at $\mathrm{f}=0.6095 \mathrm{c} / \mathrm{a}$. (c) Upward and downward radiation spectra and their ratio. The inset shows the plot of $\operatorname{Re}\left[\mathrm{E}_{\mathrm{z}}\right]$.

In order to confirm the unidirectional laser performance, the broadband dipole sources of TE polarization were located at the position of the thin gain medium layer, the middle of the slab, and the optical powers (Poynting vectors) radiating in the upward and the downward were calculated at the locations near the upper and the lower absorbing boundaries, which are plotted in Fig. 4(c). The ratio between the upward and the downward radiation is $\sim 4.6 \times 10^{4}$ at the resonant frequency. The inset 
shows the field distribution at the resonant frequency, confirming the almost unidirectional radiation into the upward direction, where the real part of $E_{z}$ is plotted to show the wave propagation clearly.

\section{CONCLUSION}

In conclusion, we proposed a novel approach to implement a mirror-less unidirectional surface emitting laser based on an intuitive and simple concept of mimicking one-port system with a single asymmetric resonator of two degenerate modes. In the proposed approach, one of the resonant modes combined with the background scattering serves as an internal reflector, and the other one serves as a lasing mode. The operating principle of the proposed unidirectional laser has been analyzed with the TCMT, which reveals that a passive structure designed to have $100 \%$ reflection at the resonant frequency with a proper coupling strength between two degenerate modes through the background scattering works as the unidirectional laser with introduction of proper optical gain. An example device has been designed based on two GMR modes in a ZCG. The design approach has been confirmed by the RCWA calculation, and the performance of the designed device has been analyzed with the FDTD simulation, demonstrating the ratio of $4.6 \times 10^{4}$ between the upward and the downward radiations.

Although we focused on the unidirectional radiation application of the proposed scheme in this work, from a viewpoint of the time-reversal symmetry, it can also be applied for perfect absorption under single-sided illumination. Therefore, we believe that our proposed scheme paves a new pathway toward the control of the directionality of a single resonator for various useful device application.

\section{APPENDIX}

Indirect coupling between two resonant modes induced by partial reflection in a two-port asymmetric optical resonator

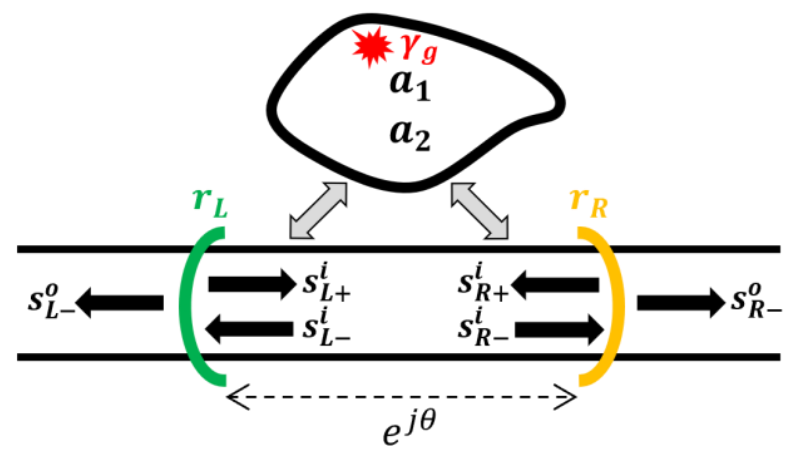

Fig. A1. Schematic of two-port asymmetric optical resonator of two resonant modes for mutual coupling coefficient calculation considering partial reflection in the wave propagation channel.

Fig. A1 shows the schematic of two-port asymmetric optical resonator of two resonant modes for the mutual coupling coefficient calculation considering the partial reflection in the wave propagation channel, which is basically the same as Fig. 1. Now, we focus on the interaction between the resonant modes and the internal waves propagating in the channel cavity formed by two partial reflections $\left(r_{L}, r_{R}\right)$, assuming no incoming waves into the cavity. Two resonant modes $\left(a_{1}, a_{2}\right)$ decays into the channel cavity with decay rates of $\frac{1}{\tau_{1}}$ and $\frac{1}{\tau_{2}}$, respectively, and are excited by internal waves propagating toward the resonator $\left(s_{L+}^{i}, s_{R+}^{i}\right)$ with the corresponding coupling coefficients $\left(\kappa_{L 1}^{\prime}, \kappa_{L 2}^{\prime}, \kappa_{R 1}^{\prime}, \kappa_{R 2}^{\prime}\right)$. The rate equations are given by

$$
\begin{gathered}
\frac{d a_{1}}{d t}=\left(j \omega_{1}-\frac{1}{\tau_{1}}+\gamma_{g}\right) a_{1}+\kappa_{L 1}^{\prime} \cdot s_{L+}^{i}+\kappa_{R 1}^{\prime} \cdot s_{R+}^{i}, \\
\frac{d a_{2}}{d t}=\left(j \omega_{2}-\frac{1}{\tau_{2}}\right) a_{2}+\kappa_{L 2}^{\prime} \cdot s_{L+}^{i}+\kappa_{R 2}^{\prime} \cdot s_{R+}^{i},
\end{gathered}
$$

Note that in (A-1), two resonant modes do not interact directly, and they interact with each other via the internal waves. The internal waves propagation toward the ports $\left(s_{L-}^{i}, s_{R-}^{i}\right)$ are related with the internal waves propagating toward the resonator $\left(s_{L+}^{i}, s_{R+}^{i}\right)$ and the resonant mode amplitudes as follows:

$$
\begin{aligned}
& s_{L-}^{i}=e^{-j \theta} \cdot s_{R+}^{i}+d_{L 1}^{\prime} \cdot a_{1}+d_{L 2}^{\prime} \cdot a_{2}, \\
& s_{R-}^{i}=e^{-j \theta} \cdot s_{L+}^{i}+d_{R 1}^{\prime} \cdot a_{1}+d_{R 2}^{\prime} \cdot a_{2},
\end{aligned}
$$

where $d_{P i}^{\prime}$ is the coupling coefficient of i-th mode into the internal wave propagating toward P-port. Note that the coupling coefficients in (A-1) and (A-2) are different from those in section 2, and this is why the different notation is used here. The internal wave propagating toward the resonator are also related to the internal waves propagating toward the port via the partial reflections $\left(r_{L}, r_{R}\right)$ at the respective ports, which are given by

$$
\begin{aligned}
& L-\text { port }: s_{L+}^{i}=r_{L} \cdot s_{L-}^{i} \\
& R-\text { port }: s_{R+}^{i}=r_{R} \cdot s_{R-}^{i}
\end{aligned}
$$

Combining (A-2) and (A-3), we obtain

$$
\begin{aligned}
& s_{L+}^{i}=r_{L} \cdot\left(e^{-j \theta} \cdot s_{R+}^{i}+d_{L 1}^{\prime} \cdot a_{1}+d_{L 2}^{\prime} \cdot a_{2}\right), \\
& s_{R+}^{i}=r_{R} \cdot\left(e^{-j \theta} \cdot s_{L+}^{i}+d_{R 1}^{\prime} \cdot a_{1}+d_{R 2}^{\prime} \cdot a_{2}\right) .
\end{aligned}
$$

From (A-4), the internal waves propagation toward the resonator $\left(s_{L+}^{i}, s_{R+}^{i}\right)$ can expressed in terms of the mode amplitudes $\left(a_{1}, a_{2}\right)$ as follows:

$$
\begin{array}{r}
s_{L+}^{i}=\frac{1}{1-r_{L} \cdot r_{R} \cdot e^{-j 2 \theta}}\left[\left(r_{L} \cdot r_{R} \cdot d_{R 1}^{\prime} \cdot e^{-j \theta}+r_{L} \cdot d_{L 1}^{\prime}\right) \cdot a_{1}+\right. \\
\left.\left(r_{L} \cdot r_{R} \cdot d_{R 2}^{\prime} \cdot e^{-j \theta}+r_{L} \cdot d_{L 2}^{\prime}\right) \cdot a_{2}\right], \quad(\mathrm{A}-5 \mathrm{a}) \\
s_{R+}^{i}=\frac{1}{1-r_{L} \cdot r_{R} \cdot e^{-j 2 \theta}}\left[\left(r_{L} \cdot r_{R} \cdot d_{L 1}^{\prime} \cdot e^{-j \theta}+r_{R} \cdot d_{R 1}^{\prime}\right) \cdot a_{1}+\right. \\
\left.\left(r_{L} \cdot r_{R} \cdot d_{L 2}^{\prime} \cdot e^{-j \theta}+r_{R} \cdot d_{R 2}^{\prime}\right) \cdot a_{2}\right] .(\mathrm{A}-5 \mathrm{~b})
\end{array}
$$

By substituting (A-5) into (A-1), we obtain

$$
\begin{array}{r}
\frac{d a_{1}}{d t}=\left(j \omega_{1}-\frac{1}{\tau_{1}}+\lambda_{1}+\gamma_{g}\right) a_{1}+\mu_{12} \cdot a_{2} \\
\frac{d a_{2}}{d t}=\left(j \omega_{1}-\frac{1}{\tau_{2}}+\lambda_{2}\right) a_{2}+\mu_{21} \cdot a_{1}
\end{array}
$$

where 


$$
\begin{aligned}
& \lambda_{1}=\frac{1}{1-r_{L} \cdot r_{R} \cdot e^{-j 2 \theta}}\left(r_{L} \cdot d_{L 1}^{\prime 2}+r_{R} \cdot d_{R 1}^{\prime 2}+2 r_{L} \cdot r_{R} \cdot d_{L 1}^{\prime} \cdot d_{R 1}^{\prime} .\right. \\
& \left.e^{-j \theta}\right), \quad(\mathrm{A}-6 \mathrm{c}) \\
& \lambda_{2}=\frac{1}{1-r_{L} \cdot r_{R} \cdot e^{-j 2 \theta}}\left(r_{L} \cdot d_{L 2}^{\prime 2}+r_{R} \cdot d_{R 2}^{\prime 2}+2 r_{L} \cdot r_{R} \cdot d_{L 2}^{\prime} \cdot d_{R 2}^{\prime} .\right. \\
& \left.e^{-j \theta}\right) \text {, } \\
& \left.\left.d_{R 1}^{\prime}\right) \cdot e^{-j \theta}+r_{L} \cdot d_{L 1}^{\prime} \cdot d_{L 2}^{\prime}+r_{R} \cdot d_{R 1}^{\prime} \cdot d_{R 2}^{\prime}\right) . \quad(\mathrm{A}-6 \mathrm{e})
\end{aligned}
$$

In driving (A-6c)-(A6e), the relations of $\kappa_{L 1,2}^{\prime}=d_{L 1,2}^{\prime}$ and $\kappa_{R 1,2}^{\prime}=d_{R 1,2}^{\prime}$ are also used.

As seen in (A-6e), the mutual coupling coefficients are the same. $\lambda_{1}$ and $\lambda_{2}$ are the coefficients representing the resonance frequency and decay rate adjustment due to the channel cavity. Note that $\mu=\mu_{12}=\mu_{21}, \lambda_{1}$ and $\lambda_{2}$ are complex in general, and the decay rate in (1) can be related to $\lambda_{1}$ as $\gamma_{i}=\frac{1}{\tau_{i}}-\lambda_{i}$.

From these results, we can reformulate (1) with $\Omega_{m}$ and $\Gamma_{m}$ defined as

$$
\Omega_{m}=\left(\begin{array}{cc}
\omega_{1}+j \frac{\gamma_{1}-\gamma_{1}^{*}}{2} & 0 \\
0 & \omega_{2}+j \frac{\gamma_{2}-\gamma_{2}^{*}}{2}
\end{array}\right), \Gamma_{m}=\left(\begin{array}{cc}
\frac{\gamma_{1}+\gamma_{1}^{*}}{2} & \mu \\
\mu & \frac{\gamma_{2}+\gamma_{2}^{*}}{2}
\end{array}\right) .
$$

The imaginary part of the complex decay rate shifts the resonant frequency $\left(\omega_{i}\right)$ to the effective resonance frequency $\left(\omega_{i}+j \frac{\gamma_{i}-\gamma_{i}^{*}}{2}\right)$. The real part of the complex decay rate represents the modal energy decay as seen in (7a) and (7b).

\section{REFERENCES}

[1] K.X. Wang, Z. Yu, S. Sandhu and S. Fan, '"Fundamental bounds on decay rates in asymmetric single-mode optical resonators," Opt.Lett., vol. 38, no. 2, pp. 100-102.

[2] B. Ben Bakir et al., "Surface-emitting microlaser combining twodimensional photonic crystal membrane and vertical Bragg mirror," Appl.Phys.Lett., vol. 88, no. 8, p. 081113, 2006.

[3] H. C. Lin, K. L. Chang, K. C. Hsieh, K. Y. Cheng, and W. H. Wang, "Metallic wafer bonding for the fabrication of long-wavelength verticalcavity surface-emitting lasers," J.Appl.Phys., vol. 92, no. 7, pp. 41324134, 2002.

[4] H. Zhou et al., "Perfect single-sided radiation and absorption without mirrors," Optica, vol. 3, no. 10, pp. 1079-1086, 2016.

[5] H. Heo, S. Lee, and S. Kim, "Tailoring fano resonance for flat-top broadband reflectors based on single guided-mode resonance," J.Lightwave Technol., vol. 37, no. 17, pp. 4244-4250, 2019.

[6] W. Suh, Z. Wang, and S. Fan, "Temporal coupled-mode theory and the presence of non-orthogonal modes in lossless multimode cavities," IEEE J.Quant.Electron., vol. 40, no. 10, pp. 1511-1518, 2004.

[7] R. Magnusson, "Wideband reflectors with zero-contrast gratings," Opt.Lett., vol. 39, no. 15, pp. 4337-4340, 2014.

[8] M. G. Moharam, E. B. Grann, D. A. Pommet, and T. K. Gaylord, "Formulation for stable and efficient implementation of the rigorous coupled-wave analysis of binary gratings," JOSA $a$, vol. 12, no. 5, pp. 1068-1076, 1995
[9] S. Fan and J. D. Joannopoulos, "Analysis of guided resonances in photonic crystal slabs," Physical Review B, vol. 65, no. 23, p. 235112, 2002.

Joohyung Song received the B.S. degree from the Department of Electrical and Computer Engineering, Ajou University, Suwon, South Korea, where he is currently working toward the master's degree. His research interests include optical fiber, photonic crystals, guided mode resonance, and coupled mode theory.

Hyungjun Heo received the Ph.D. degree from the Department of Electrical and Computer Engineering, Ajou University, Suwon, South Korea, in 2019. His doctoral works are involved in the design of optical device based on theoretical modeling. Since 2020, he has been a Postdoctoral Fellow at the center of quantum information, Korea Institute of Science and Technology, Seoul, South Korea, where he has been responsible for the design of integrated quantum photonic device. His research interests include advanced photonic materials, quantum information, photonic integrated circuits, guided mode resonance, and coupled mode theory.

Sangjun Lee received the Ph.D. degree from the Department of Electrical and Computer Engineering, Ajou University, Suwon, South Korea, in 2011. His doctoral works are involved in the design of novel plasmonic waveguide structures. Between 2011 and 2014, he was a Postdoctoral Fellow with the Photonic Systems Laboratory, Seoul National University, Seoul, South Korea, where he has been responsible for the ultrathin solar cell design. Since 2015, he has been a Research Assistant Professor with Ajou University. His main research interests include graphene plasmonics, graphene perfect absorbers, plasmonics solar cells, optical sensor, optical phased arrays, hologram, high-contrast gratings, and metamaterials.

Sangin Kim received the B.S. degree in electrical engineering from the Korea Advanced Institute of Science and Technology (KAIST), Daejeon, South Korea, in 1992, and the M.S. and $\mathrm{Ph} . \mathrm{D}$. degrees in electrical engineering from the University of Minnesota, Minneapolis-St. Paul, MN, USA, in 1995 and 1997, respectively. From 1997 to 2002, he was with Korea Telecom, where he was involved in works on high-capacity optical switching systems, optical access networks, and polarization mode dispersion compensation for high bit rate optical transmission. In 2003, he joined the Department of Electrical and Computer Engineering, Ajou University, Suwon, South Korea, where he is currently a Professor. His research interest covers broad spectrum of nanophotonics, nanolasers, guided wave optics, plasmonics, photonic integrated circuits, and quantum information. 